Available on line at www.rac.es/racsam Mathematical Analysis

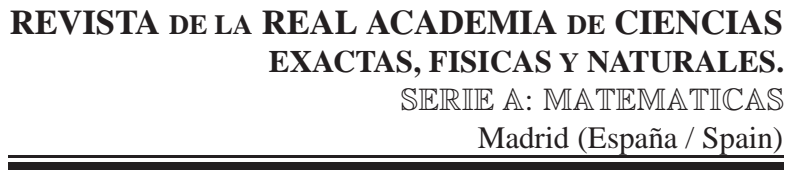

RACSAM 104 (1), 2010, 13-18. DOI:10.5052/RACSAM.2010.03

\title{
$P$-spaces and an unconditional closed graph theorem
}

\author{
Marek Wójtowicz and Waldemar Sieg
}

\begin{abstract}
Let $X$ be a completely regular (Tychonoff) space, and let $C(X), U(X)$, and $B_{1}(X)$ denote the sets of all real-valued functions on $X$ that are continuous, have a closed graph, and of the first Baire class, respectively.

We prove that $U(X)=C(X)$ if and only if $X$ is a $P$-space (i.e., every $G_{\delta}$-subset of $X$ is open) if and only if $B_{1}(X)=U(X)$. This extends a list of equivalences obtained earlier by Gillman and Henriksen, Onuchic, and Iséki. The first equivalence can be regarded as an unconditional closed graph theorem; it implies that if $X$ is perfectly normal or first countable (e.g., metrizable), or locally compact, then there exist discontinuous functions on $X$ with a closed graph. This complements earlier results by Doboš and Baggs on discontinuity of closed graph functions.
\end{abstract}

\section{$P$-espacios y un teorema incondicional de gráfica cerrada}

Resumen. Sea $X$ un espacio completamente regular (Tychonoff). Por $C(X), U(X)$ y $B_{1}(X)$ se denotan los conjuntos de funciones reales definidas en $X$ que son continuas, que tienen gráfica cerrada y que son de primera clase de Baire, respectivamente. Se prueba que $U(X)=C(X)$ si y sólo si $X$ es un $P$ espacio (es decir que cada subconjunot $G_{\delta}$ de $X$ es abierto) o si y sólo si $B_{1}(X)=U(X)$. Estos resultados extienden una relación de equivalencias obtenidas por Gillman y Henriksen, Onuchic e Iséki. La primera equivalencia es un teorema incondicional de gráfica cerrada; implica que si $X$ es perfectamente normal o cumple el primer axioma de numerabilidad (por ejemplo si es metrizable), o es localmente compacto, entonces existen funciones discontinuas en $X$ con gráfica cerrada. Así se complementan resultados obtenidos por Dobos y Bags sobre discontinuidad de funciones con gráfica cerrada.

\section{Introduction}

Throughout this paper $X, Y$ denote Hausdorff spaces, $C(X), U(X)$, and $B_{1}(X)$ have the same meanings as in the Abstract, and $\mathbb{R}$ denotes the set of real numbers endowed with the natural topology. If $f$ is a function on $X$, then $D(f)$ denotes the set of discontinuous points of $f$; hence $C(f):=X \backslash D(f)$ is the set of continuity points of $f$.

Submitted by Manuel Valdivia

Received: November 20, 2009. Accepted: January 13, 2010

Keywords: Real-valued functions with a closed graph, points of discontinuity, completely regular or $P$-spaces, Baire functions Mathematics Subject Classifications: Primary 26A15, 54D10; secondary 54C10, 54C30

(c) 2010 Real Academia de Ciencias, España 
The study of discontinuous closed graph functions was initiated in 1974 by Ivan Baggs [1]. He showed that if $X=\mathbb{R}$ then, for every closed and nowhere dense subset $F$ of $X$, there is a function $f \in U(X)$ such that $D(f)=F$. In 1985 Doboš [7, Theorems 7 and C] generalized Baggs's result to two cases: for $X$ a perfectly normal space and $F$ of first category in $X$, and for $X$ a metric Baire (e.g., complete) space and $F$ closed and nowhere dense in $X$. In 1989 Baggs considered a similar problem under a weaker assumption on $X$ : he showed in [3, Theorem 4.3] that if $X$ is completely regular then, given $F$ a compact $G_{\delta}$ and of first category subset of $X$, the equation $D(f)=F$ has a solution $f \in U(X)$. The above-cited results led obviously to the natural question:

Whether there exists a space $X$ such that every closed graph function on $X$ is continuous, i.e., if $U(X)=C(X)$ ?

In 1989 Baggs [2, Example 4.2] showed this question has a positive answer: he has constructed a regular (yet non-completely regular) space $X$ on which every real-valued function with a closed graph is constant; hence

$$
U(X)=C(X)=B_{1}(X) .
$$

It is worth to add that Baggs [2, Example 5.2] has also given an example of a regular and non-completely regular space $Y$ such that $C(Y)=B_{1}(Y)$, yet $U(Y) \neq C(Y)$.

The purpose of this paper is to give a full characterization of equations (1) for $X$ a completely regular space (Theorem 1 below). In particular, the characterization implies that the phenomenon described in the latter example by Baggs does not hold whenever $X$ is merely completely regular. We recall [12, pp. 62-63] that such a space $X$ is said to be a $P$-space if every $G_{\delta}$-subset [ $F_{\sigma}$-subset] of $X$ is open [closed]; equivalently, every co-zero subset of $X$ is closed (the examples of such spaces are included in the monograph by Gillman and Jerison [12, Examples 4JKL, 4N], and in the papers [8, 18, 23, 26, 28]; $P$-spaces illustrate also the essentiality of the notion of Dedekind $\sigma$-completeness of $C(X)$ [20, Theorem 43.8]).

Our main result reads as follows (its proof is given in Section 3 ).

Theorem 1 Let $X$ be a completely regular space. Then the following four conditions are equivalent:

(i) $U(X)=C(X)$;

(ii) $B_{1}(X)=C(X)$;

(iii) $B_{1}(X)=U(X)$;

(iv) $X$ is a $P$-space.

Theorem 1 extends the lists of equivalent conditions for $X$ to be a $P$-space obtained by Gillman and Henriksen [11, Theorem 5.3] (cf. [12, 4J]), and Tucker [30, Theorem 5].

The above equivalence (i) $\Longleftrightarrow$ (iv) may be regarded as an unconditional closed graph theorem (CGT) (here a conditional CGT is understood as: a function $f$ is continuous on $X$ iff $f$ has a closed graph + an extra condition on $f$ ). Conditional CGTs (in a larger setting, where the targeted space $\mathbb{R}$ is replaced by a topological space $Y$ ) were examined by a number of authors. The most known such a result is the linear Banach's CGT (see [4, Chapter 6] for a survey of its generalizations, cf. [34]), and its versions for topological groups were studied by Grant [13], Husain [14], and Wilhelm [33]. Purely topological conditional CGTs were obtianed by Fuller [10], Piotrowski and Szymański [27], Wilhelm [32], and Moors [22].

The equivalence (ii) $\Longleftrightarrow$ (iv) in Theorem 1 is a characterization of those $C(X)$-spaces, for $X$ completely regular, for which the Baire order is zero (cf. [21, p. 418 and Corollary 4.1]). This equivalence was obtained independently in 1957 by Onuchic [25], and in 1958 by Iséki [15]; it is also included implicitly in Theorem 4 by Ohno [24], and appears (without any reference) on p. 562 of Tucker's paper [30].

Remark 1 The equivalence (i) $\Longleftrightarrow$ (ii) and theorems on extensions of Baire-one functions [16, 19, 29] suggest we can deduce the existence of discontonuous closed graph functions on $X$ from the relation 
$B_{1}(A) \neq C(A)$ for a proper subset $A$ of $X$ (see, e.g., [21, pp. 431-441]). However, this method (i.e., an appeal to extension theorems) of verification of the relation $U(X) \neq C(X)$ seems to be improper, because from the equivalence (i) $\Longleftrightarrow$ (iv) of Theorem 1 and property [12, 4K(4)] (that every subspace of a $P$-space is a P-space again) it follows it is enough only to check if the subspace $A$ is not a $P$-space (and in a few typical cases even if $A$ is non-discrete: see Corollaries 1 and 2 below).

Since every singleton of a perfectly normal or first countable space $X$ is a $G_{\delta}$-set, every such a $P$-space is discrete. Moreover, every locally compact $P$-space is discrete too (this follows easily from [12, 4K(3)]). Hence Theorem 1 has the following immediate consequence.

Corollary 1 Let $X$ be a perfectly normal or first countable space (e.g., metrizable), or a locally compact space. Then the following four conditions are equivalent:

(i) $U(X) \neq C(X)$;

(ii) $B_{1}(X) \neq C(X)$;

(iii) $B_{1}(X) \neq U(X)$;

(iv) $X$ is non-discrete.

The next corollary follows from the fact that every $P$-space is basically disconnected [12, 4K(7)] (the latter means that every co-zero subset of $X$ has an open closure).

Corollary 2 Let $X$ be a completely regular space. If, additionally, $X$ is connected, then there exist discontinuous closed graph functions on $X$.

The last corollary deals with two spaces of functions of the first Baire class. A function $f: X \rightarrow \mathbf{R}$ is said to be

- piecewise continuous if there is a sequence $\left(X_{n}\right)$ of closed subsets of $X$ such that $X=\bigcup_{n=1}^{\infty} X_{n}$ and the restrictions $F_{\mid X_{n}}$ are continuous for all $n$ 's;

- Baire-one-star function if for every closed subset $F$ of $X$ the set $C\left(f_{\mid F}\right)$ has nonempty interior (in the induced topology on $F$ ).

The linear spaces of piecewise continuous and Baire-one-star functions on $X$ are denoted by $\mathcal{P}(X)$ and $B_{1}^{*}(X)$, respectively. These two spaces were studied by Borsik [5], Borsik, Doboš and Repický [6], and Kirchheim [17], among others.

It is known that $U(X) \subset \mathcal{P}(X)$ for $X$ arbitrary [5, p. 119], that $\mathcal{P}(X)=U(X)+U(X)$ for $X$ is perfectly normal [5, Theorem 1], and that $\mathcal{P}(X)=B_{1}^{*}(X)$ for $X$ a complete metric space [17, Theorem 2.3]. It is easy to check that if $X$ is a $P$-space, then every piecewise continuous function $f$ on $X$ is continuous (this follows from the equality $f^{-1}(F)=\bigcup_{n=1}^{\infty}\left(f_{\mid X_{n}}\right)^{-1}(F)$, where $X=\bigcup_{n=1}^{\infty} X_{n}$ ). Moreover, $U(X) \subset \mathcal{P}(X)$ (because every $f \in U(X)$ is continuous on the closed set $X_{n}:=f^{-1}[-n, n]$ ). Hence, by Theorem 1 (i), we obtain yet another characterization of $P$-spaces:

Corollary 3 Let $X$ be a completely regular space. Then $X$ is a P-space if and only if $\mathcal{P}(X)=C(X)$. In particular, if $X$ is a complete metric space, then $B_{1}^{*}(X) \neq C(X)$ if and only if $X$ is non-discrete.

Remark 2 Looking at Theorem 1 we can conjecture that the equality $\mathcal{P}(X)=B_{1}(X)$ should imply $X$ to be a $P$-space, but this is not the case. Indeed, let $X$ denote the set of all rational numbers endowed with the natural (metric) topology. Since $X$ is a metric space, from the Tietze theorem we get $\mathcal{P}(X) \subset B_{1}(X)$. It is also easy to check that $\mathcal{P}(X)=\mathbb{R}^{X}$, whence $\mathcal{P}(X)=B_{1}(X)$. On the other hand, by [12, $\left.4 \mathrm{~K}(1)\right]$ (that every countable $P$-space is discrete), $X$ is not a $P$-space. 


\section{Notations}

For the basic facts concerning topology and continuous functions we refer the reader to the monographs [ 9 , 12]. We recall that a subset $A$ of $X$ is said to be a zero-set if there is a continuous function $f: X \rightarrow[0,1]$ such that $A=[f=0]:=f^{-1}(0)$; then the set $[f>0]:=X \backslash A$ is called co-zero. The symbol $\operatorname{Fr}(A)$ denotes the frontier of $A$, i.e., $\operatorname{Fr}(A):=\bar{A} \cap \overline{X \backslash A}=\bar{A} \backslash \operatorname{Int}(A)$, where $\bar{A}$ is the closure of $A$, $\operatorname{and} \operatorname{Int}(A)$ denotes the interior of $A$.

\section{The proof of Theorem 1}

The proof of Theorem 1 is based on a few properties of continuous and closed graph functions collected in Lemmas 1 and 2 below.

We start with a result generalizing the constructions of Doboš [7] and Baggs [3] of discontinuous closed graph functions. The reader should note that in our Lemma 1 the (nonempty) zero-set $[f=0]$ is arbitrary, while the above-mentioned authors assume it to be nowhere dense [7, Theorem 5], or compact [3, Theorem 3.2, Theorem 4.3].

Lemma 1 Let $f: X \rightarrow[0,1]$ be a continuous function, and let the set $[f=0]$ be not empty. Consider the function

$$
f^{*}(x)= \begin{cases}\frac{1}{f(x)} & \text { if } x \in[f>0], \\ 0 & \text { if } x \in[f=0] .\end{cases}
$$

Then $f^{*}$ has a closed graph, and $D\left(f^{*}\right)=\operatorname{Fr}([f=0])$.

Proof. A proof that $f^{*} \in U(X)$ is actually due to Doboš [7, Proof of Theorem 5]; Baggs [1, Proof of Theorem 4.3] applies elementary arguments: define $\phi: \mathbb{R} \rightarrow \mathbb{R}$ as $\phi(0)=0$ and $\phi(x)=1 / x$ otherwise, notice that $\phi \in U(\mathbb{R})$, and that the composition $\phi \circ f$ equals $f^{*}$, whence $f^{*} \in U(X)$.

Now we prove that $D\left(f^{*}\right)=\operatorname{Fr}([f=0])$; equivalently,

$$
C\left(f^{*}\right)=[f>0] \cup \operatorname{Int}([f=0]) .
$$

Since the both sets on the right side of (2) are open and $f^{*}$ is continuous on each of them, the inclusion $\supseteq$ in (2) is obvious.

On the other hand,

iff $x_{0} \in \operatorname{Fr}([f=0])$; hence

$$
x_{0} \notin[f>0] \cup \operatorname{Int}([f=0])
$$

$$
f^{*}\left(x_{0}\right)=0=f\left(x_{0}\right)
$$

Let us fix a basis $\mathcal{V}\left(x_{0}\right)$ of neighbourhoods of such an $x_{0}$. Since

$$
x_{0} \in \overline{[f>0]} \supset \operatorname{Fr}([f=0]),
$$

for every $V \in \mathcal{V}\left(x_{0}\right)$ there is $y_{V} \in V$ such that

$$
f\left(y_{V}\right)>0
$$

From (4) we obtain

$$
f^{*}\left(y_{V}\right)=1 / f\left(y_{V}\right) \geq 1 .
$$

Hence, by (3) and (4), for every $V \in \mathcal{V}\left(x_{0}\right)$ there is $y \in V$ such that

$$
\left|f^{*}(y)-f^{*}\left(x_{0}\right)\right| \geq 1 \text {. }
$$

Therefore $x_{0} \in D\left(f^{*}\right)$, i.e., $x_{0} \notin C\left(f^{*}\right)$. We thus have proved the inclusion $\subseteq$ in (2) is also true. The proof of Lemma 1 is complete.

In the proof of Theorem 1 we shall apply the following three properties. 
Lemma 2 Let $X$ be a Hausdorff space.

(a) If $X$ is a $P$-space and $\xi \in X$, then every function $f \in C(X)$ is constant on a neighborhood of $\xi[12$, $4 \mathrm{~J}(2)]$.

(b) Let $f \in U(X)$. Then (see [31, p. 196 - Facts (iii) and (iv)])

(*) for every closed interval $[a, b]$ the set $f^{-1}[a, b]$ is closed;

(**) if $f$ is bounded, it is continuous.

\section{PROOF OF THEOREM 1.}

Non-(iv) implies non-(i). Since $X$ is not a $P$-space, there is a continuous function $f$ on $X$ such that the zero set $[f=0]$ is non-open. The latter implies that the set $\operatorname{Fr}([f=0])$ is not empty. By Lemma 1, the function $f^{*}$ has a closed graph and $D\left(f^{*}\right) \neq \emptyset$, i.e., $f^{*}$ is discontinuous. Hence $U(X) \neq C(X)$.

Both (ii) and (iii) implies (iv). Set $U=[f>0]$, where $f$ is an arbitrary fixed element of $C(X)$. It is known (see [16, Proof of Proposition 2]) that the characteristic function $\chi_{U}$ of $U$ belongs to $B_{1}(X)$. In case (ii) we obtain that $U$ is closed, whence (as $f$ were arbitrary) $X$ is a $P$-space. In case (iii), by Lemma $2(\mathrm{~b})(* *), \chi_{U}$ is continuous, and so, as previously, $X$ is a $P$-space.

(iv) implies (ii). This is a simple consequence of Lemma 2(a): the pointwise limit $f$ of a sequence of functions $\left(f_{n}\right) \subset C(X)$, each constant on a neighborhood $V_{n}$ of $\xi \in X$, is constant on their intersection $V$, which is open by the definition of a $P$-space. Hence $f$ is continuous at $\xi$.

(iv) implies (i). Let $f \in U(X)$. We claim that, for every open interval $(a, b)$, the set $f^{-1}(a, b)$ is $G_{\delta}$, hence open by the definition of a $P$-space. By Lemma $2(\mathrm{~b})(*)$, every set $A_{n}=f^{-1}[b, b+n], n=1$, $2, \ldots$ is closed, whence the set $f^{-1}[b, \infty)=\bigcup_{n=1}^{\infty} A_{n}$ is $F_{\sigma}$. Similarly, the set $f^{-1}(-\infty, a]$ is $F_{\sigma}$ too. Consequently, $f^{-1}(a, b)$ is $G_{\delta}$, as claimed.

(iv) implies (iii). Since, as we have already showed, (iv) implies both (i) and (ii), we obtain that condition (iv) implies further that $U(X)=C(X)=B_{1}(X)$.

The proof of Theorem 1 is complete.

\section{References}

[1] Baggs, I., (1974). Functions with a closed graph, Proc. Amer. Math. Soc., 43, 2, 439-442. DOI: 10.2307 /2038910

[2] BAggs, I., (1989). A regular space on which every real-valued function with a closed graph is constant, Can. Math. Bull., 32, 417-424.

[3] Baggs, I., (1989). Nowhere dense sets and real-valued functions with closed graphs, Int. J. Math. Math. Sci., 12, 1, 1-8. DOI: 10.1155/S0161171289000013

[4] Beattie, R. And Butzmann, H.-P., (2002). Convergence structures and applications to functional analysis, Kluwer Academic Publishers, Dortrecht.

[5] Borsik, J., (2002). Sums, differences, products and quotients of closed graph functions, Tatra Mt. Math. Publ., 24, 117-123.

[6] Borsik, J.; Doboš, J. AND REPICKÝ, M., (1999/2000). Sums of quasicontinouos functions with closed graphs, Real Anal. Exchange, 25, 679-690.

[7] Doвoš, J., (1985). On the set of points of discontinuity for functions with closed graphs, Cas. Pěst. Mat., 110, $60-68$.

[8] Dow, A., (1983). On F-spaces and $F^{\prime}$-spaces, Pacific J. Math., 108, 275-284.

[9] Engelking, R., (1989). General Topology, Helderman-Verlag, Berlin.

[10] Fuller, R. V., (1968). Relations among continuous and various non-continuous functions, Pacific J. Math., 25, 495-509. 
[11] Gillman, L. And Henriksen, M., (1954). Concerning rings of continuous functions, Trans. Amer. Math. Soc., 77, 2, 340-362. DOI: 10.2307/1990875

[12] Gillman,L. And Jerison, M., (1976). Rings of Continuous Functions, Springer-Verlag, Berlin.

[13] GRAnT, D. L., (1977). Topological groups which satisfy an open mapping theorem, Pacific J. Math., 68, 411423.

[14] Husain, T., (1968). On a Closed Graph Theorem for Topological Groups, Proc. Japan Acad., 44, 6, 446-448. DOI: $10.3792 / \mathrm{pja} / 1195521147$

[15] IsÉKi, K., (1958). A Characterisation of P-spaces, Proc. Japan Acad., 34, 7, 418-419. DOI: 10.3792/pja $/ 1195524602$

[16] Kalenda, O. And Spurny, J., (2005). Extending Baire-one functions on topolgical spaces, Topology Appl., 149, 1-3, 195-216. DOI: 10.1016/j.topol.2004.09.007

[17] Kirchineim, B., (1992/93). Baire one star functions, Real Anal. Exchange, 18, 385-389.

[18] Kunen, K., (1989). Rigid P-spaces, Fund. Math., 133, 59-65.

[19] KuRatowsKi, K., (1933). Sur les théorèmes topologiques de la théorie des fonctions de variables réelles, C. R. Acad. Sci. Paris, 197, 19-20.

[20] Luxemburg, W. A. J. And ZaAnen, A. C., (1971). Riesz Spaces I, North-Holland, Amsterdam.

[21] Mauldin, R. D., (1974). Baire Functions, Borel Sets, and Ordinary Function Systems, Adv. Math., 12, 4, 418450. DOI: 10.1016/S0001-8708(74)80011-3

[22] Moors, W. B., (2002). Closed graph theorems and Baire spaces, New Zealand J. Math., 31, 55-62.

[23] Nakano, H., (1941). Über das System aller stetigen Funktionen auf einen topologischen Raum, Proc. Imp. Acad. Tokyo, 17, 308-310.

[24] Ohno, T., (1985). On the Baire order problem for a linear lattice of functions, Fund. Math., 125, $209-216$.

[25] Onuchic, N., (1957). On two properties of P-spaces, Port. Math., 16, 37-39.

[26] Osba, E. A. And Henriksen, M., (2004). Essential $P$-spaces: a generalization of door spaces, Comment. Math. Univ. Carolinae, 45, 509-518.

[27] Piotrowski, Z. And Szymański, A., (1988). Closed graph theorem: Topological approach, Rend. Circ. Mat. Palermo, Ser. II, 37, 1, 88-99. DOI: 10.1007/BF02844269

[28] Raphael, R. And Woods, R. G., (2006). On RG-spaces and the regularity degree, Appl. Gen. Topol., 7 , 73-101.

[29] Shatery, H. R. And Zafarani, J., (2004/2005). The equality between Borel and Baire classes, Real Anal. Exchange, 30, 373-384.

[30] TUCKER, C. T., (1984). Pointwise and order convergence for spaces of continuous functions and spaces of Baire functions, Czech. Math. J., 34, (109), 562-569.

[31] Wilansky, A., (1964). Functional analysis, Blaisdell Publishing Company, New York.

[32] Wilhelm, M., (1979). Relations among some closed graph and open mapping theorems, Colloq. Math., 42, 387-394.

[33] Wilhelm, M., (1979). On closed graph theorems in topological spaces and groups, Fund. Math., 104, 85-95.

[34] Zhong, S.; Li, R. And Won, S. Y., (2008). An improvement of a recent closed graph theorem, Topology Appl., 155, 15, 1726-1729. DOI: 10.1016/j.topol.2008.05.012

\author{
Marek Wójtowicz \\ Instytut Matematyki, \\ Uniwersytet Kazimierza Wielkiego, \\ Pl. Weyssenhoffa 11, \\ 85-072 Bydgoszcz, \\ Poland \\ mwojteukw.edu.pl
}

\author{
Waldemar Sieg \\ Instytut Matematyki, \\ Uniwersytet Kazimierza Wielkiego, \\ Pl. Weyssenhoffa 11, \\ 85-072 Bydgoszcz, \\ Poland \\ waldeks@ukw.edu.pl
}

